\title{
Cloning of BNIP3h, a member of proapoptotic BNIP3 family genes
}

\author{
Mohammad Farooq', Younghee Kim ${ }^{1}$, \\ Sanguk Im¹, Eunjung Chung ${ }^{1}$, \\ Sunyoung Hwang ${ }^{1}$, Miyoung Sohn', \\ Moonkyu Kim ${ }^{1}$ and Jungchul Kim ${ }^{1,2}$ \\ ${ }^{1}$ Department of Immunology, Kyungpook National University School \\ of Medicine, Teagu 700-422, Korea \\ ${ }^{2}$ Corresponding author: Tel, +82-53-420-6977; \\ Fax, +82-53-423-4628; E-mail, moonkim@ @nu.ac.kr
}

Accepted 11 September, 2001

Abbreviation: BNIP-3, Bcl-2/19kda interacting protein-3; BNIP3h, $B N I P 3$ homolog; $\mathrm{BH}-3, \mathrm{Bcl}-2$ homology domain-3; TM, transmembrane domain

\begin{abstract}
Apoptosis is regulated by interaction of antiapoptotic Bcl-2 family proteins with various proapoptotic proteins, several of which are also members of the Bcl-2 family. BNIP3 (formerly NIP3) is a proapoptotic mitochondrial protein classified in the $\mathrm{Bcl}-2$ family based on limited sequence homology-3 (BH3) domain and $\mathrm{COOH}$-terminal transmembrane domain. Sequence comparison of BNIP3 has indicated that there are several BNIP3 human homologs of this protein, like BNIP3L, Nix and BNIP3. We have cloned a new member of BNIP3 family from the cDNA library prepared from human dermal papilla cells and designated as BNIP3h. BNIP3h shows substantial homology with other BNIP3 family proteins. BNIP3h induced apoptosis from $24 \mathrm{~h}$ after transfection in MCF7 cell lines and its apoptosis inducing activity is extended until $72 \mathrm{~h}$ after transfection.
\end{abstract}

Keywords: Apoptosis, BNIP3 family proteins, BNIP3h

\section{Introduction}

Apoptosis is an essential physiological process of selective elimination of cells in multicellular organism. This process is invoked during normal organ development and tissue homeostasis and also during certain pathological conditions that result in degenerative diseases. Several regulatory components of the apoptotic pathway have been identified in various living organism including man.

The process of apoptosis is also initiated as defensive mechanism in cells infected by pathogenic agents, such as viruses. Several cellular and viral proteins related to $\mathrm{Bcl}-2$ proto-oncoproteins are efficient inhibitors of apoptosis. The antiapoptotic Bcl-2 family proteins have been shown to complex with a number of cellular proteins (Reed et al., 1997). Some of these proteins themselves are also members of the Bcl-2 family. These Bcl-2 family proteins generally promote apoptosis when ectopically overexpressed. The proapoptotic Bcl-2 family proteins share one or more conserved domains with $\mathrm{Bcl}-2$ and related antiapoptosis proteins. All of the proapoptotic proteins share a common death effector domain designated $\mathrm{BH}-3$. The $\mathrm{BH}-3$ domain of proapoptotic $\mathrm{Bcl}-2$ family proteins is indispensible for the execution of cell death and for heterodimerization with antiapoptosis proteins.

Yeast two hybrid screen of proteins that interact with E1B 19K identified several unique cDNAs named NIP1, NIP2 and NIP3 (Boyd et al., 1994). All three proteins interact with discrete domains of E1B $19 \mathrm{~K}$ protein and $\mathrm{Bcl}-2$ that are involved in suppression of cell death. BNIP3 (Yasuda et al., 1998a) is a mitochondrial protein that induces apoptosis, when transiently expressed. Several homologs of the BNIP3 protein have also been reported; BNIP3L (Matsushima et al., 1998), BNIP3 $\alpha$ (Yasuda et al., 1999), Nix (Chen et al., 1999) and a BNIP3 homolog in C. elegans (Yasuda et al., 1998b). All of these proteins retain the same intrinsic proapoptotic activity like BNIP3.

It has been shown that BNIP3 also contains a $\mathrm{BH}-3$ domain (Yasuda et al., 1998a). Although most BH-3 containing proapoptosis proteins induce rapid cell death when overexpressed, BNIP3 exhibited delayed level of proapoptotic activity (Yasuda et al., 1999).

We have cloned a new member of BNIP3 from the cDNA library prepared from human dermal papilla cells and designated as BNIP3h that shows substantial homology and similar characteristics with other BNIP3 family proteins.

\section{Material and Methods}

\section{cDNA library construction and BNIP3h cloning}

Human dermal Papilla (DP) cells were cultured in Dulbecco's Eagle medium (DMEM) (Gibco BRL, Gaithersburg, MD, USA.) supplemented with penicillin (100 U/ $\mathrm{ml})$, streptomycin $(100 \mu \mathrm{g} / \mathrm{ml})$ and $10 \%$ fetal bovine 
serum (Hyclone Laboratories Inc, UT, USA). cDNA library was constructed by using Zap cDNA synthesis kit (Stratagene, La Jolla, CA, USA) with $4 \mu \mathrm{g}$ of poly $\mathrm{A}^{+}$ RNA obtained from primary cultured human dermal papilla cells. The phage library was converted into a pBluescript phagemid cDNA library by in vivo excision by the ExAssist/SOLR system (Stratagene). In order to make a hair specific EST database, random clones were selected and $5^{\prime}$ single-path sequencing reactions were performed with Sequenase ${ }^{\mathrm{TM}}$ kit (USB corporation, Cleveland, $\mathrm{OH}, \mathrm{USA}$ ) according to the manufacturer's protocol. The GenBank database was searched for homologous sequence using BLAST (Altschul et al., 1990). One of the clone (clone B764) has homology with BNIP3 family protein and we designated it BNIP3h.

\section{Northern blot analysis with multiple human tissues}

Human multiple tissue and human cancer cell line poly $\mathrm{A}^{+}$RNA blots were purchased from Clontech (East medow circle, CA, USA). To differentiate with other BNIP3 family genes, $3^{\prime}$ untranslated region of BNIP3h gene probe was made by digesting the clone B764 with Hind-III and Xho I. And it was used for hybridization in human multiple tissue northern blot analysis. Human multiple tissue and cancer cell line blots were prehybrized at $68^{\circ} \mathrm{C}$ for $30 \mathrm{~min}$ in prehybridization solution (Express hybridization solution, Clontech). After one hour of hybridization with radiolabelled BNIP3h probe, membranes were washed twice with 2XSSC/0.05\% SDS for 10 min each at room temperature and followed by two washes of $0.1 \mathrm{XSSC} / 0.1 \% \mathrm{SDS}$ at $50^{\circ} \mathrm{C}$ for 20 min each, and then membranes were subjected to exposure to film at $-70^{\circ} \mathrm{C}$ for overnight.

\section{Construction of expression plasmids}

Plasmids designed to express BNIP3h were constructed by cloning the coding region into pcDNA3.1/myc-His expression vector (Invitrogen). In order to make cloning between the EcoR I and Xho I of the expression vector, an EcoR I site at 5'-prime and an Xho I site at 3'-prime was introduced by PCR, The orientation and sequence was confirmed by sequencing the entire insert.

\section{Transient cell death assay}

Apoptosis assay was carried out in MCF7 cell line. (ATCC, Maryland, USA). MCF7 cells were cultured in Dulbecco's Eagle medium (DMEM) (Gibco BRL) supplemented with penicillin $(100 \mathrm{U} / \mathrm{ml})$, streptomycin $(100 \mu \mathrm{g} /$ $\mathrm{ml}$ ) and $10 \%$ fetal bovine serum. MCF7 cells were transiently transfected using Lipofectamine Plus reagent (Gibco $\mathrm{BRL}$ ) with pcDNA3.1/myc His- BNIP3h expression plasmid. $\beta$-Galactosidase expressing plasmids was cotransfected to check the transfection efficiency. Twenty four and forty eight hours after transfection, the cells were fixed, stained with X-Gal and microscopically examined. 100 to 200 blue color cells were microscopically scored as live (flat) and apoptotic (round) and were photographed (Yasuda et al., 1998a)

\section{Results}

\section{Isolation of $B N I P 3 h$ in human dermal papilla cells}

The B764 clone that contains BNIP3h sequence is composed of 2,144 nucleotides with an open reading frame of $660 \mathrm{bp}$ (220 amino acids) extending from an ATG codon at position 75 to a TGA stop codon at position 735. It includes 75 nucleotides in the 5'-untranslated region, and 1411 nucleotides in 3'-untranslated region (Figure 1). The sequence surrounding the potential start codon is in agreement with Kozak's rule (Kozak, 1991). Amino acid sequence from residues 134 to 145 showed a motif similar to the $\mathrm{BH}-3$ domain of $B N I P 3$

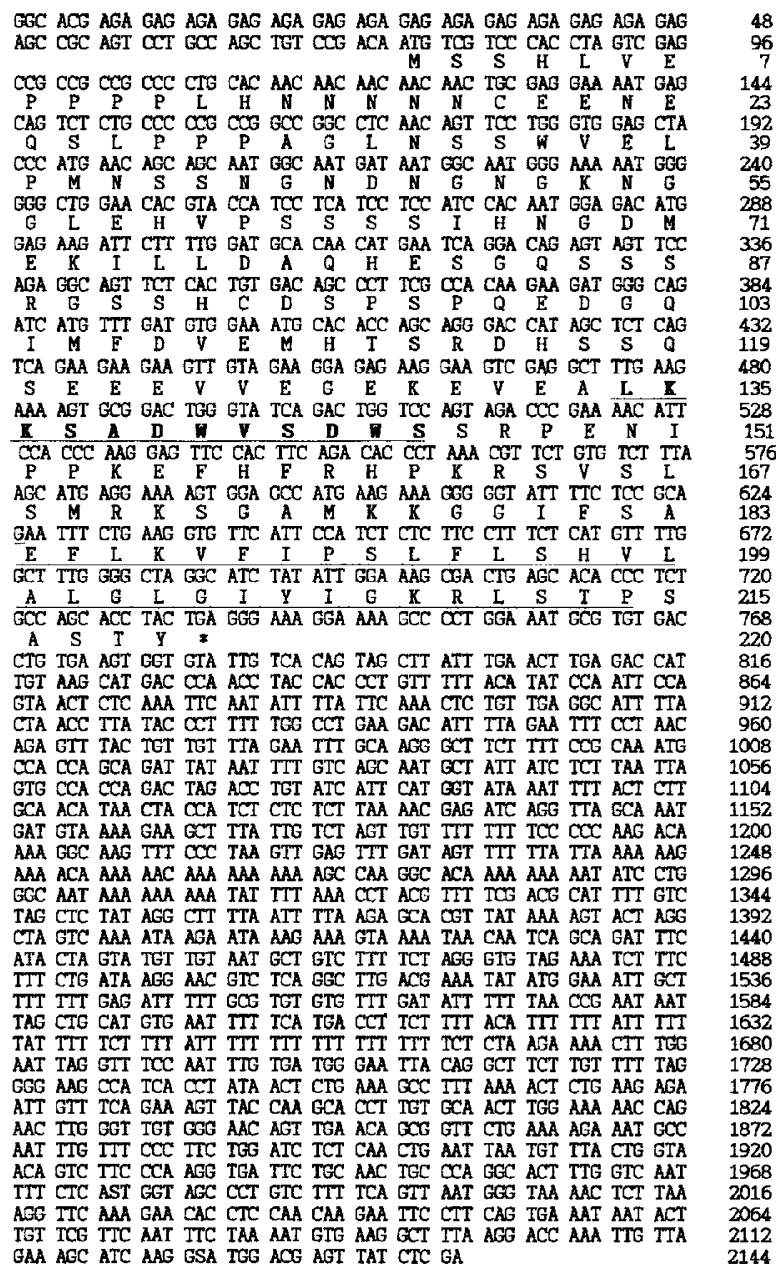

Figure 1. The nucleotide and deduced amino acids sequence of BNIP3h. Amino acids are numbered from the initiating methionine. Stop codon is indicated by an asterisk. The possible BH-3 domain is bold-underlined and transmembrane domains are underlined. 


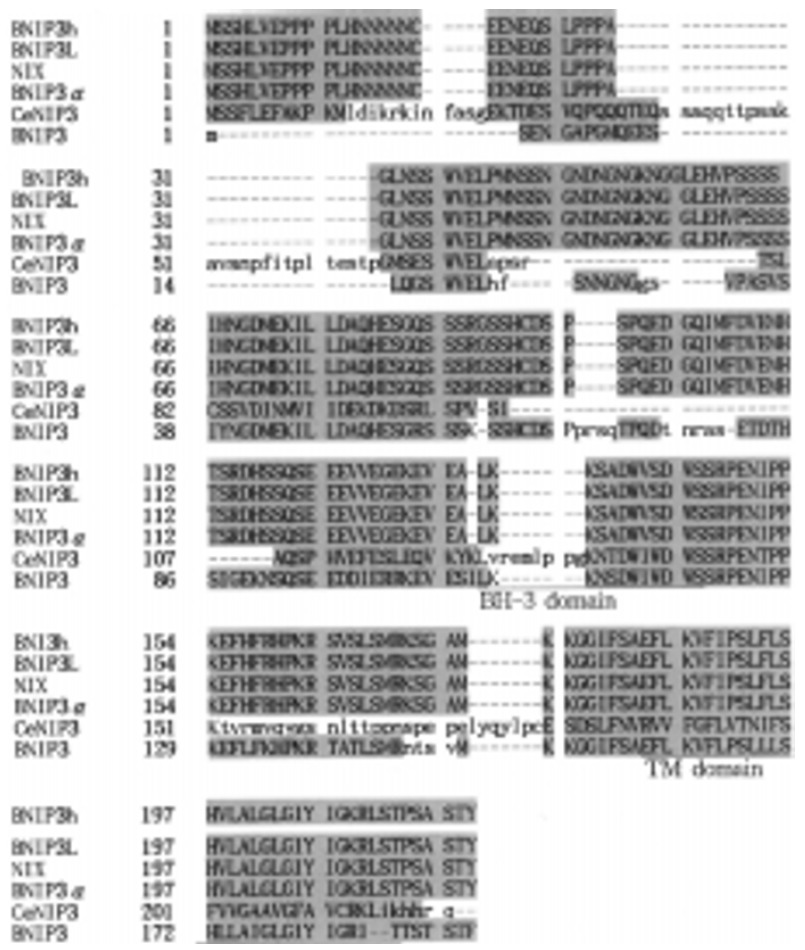

Figure 2. Identification of BNIP3h and homology to BNIP3 family proteins. Upper case letters show the identical and related amino acids between five proteins and lowercase letters show unidentical amino acids. The similar amino acids are shaded. The BH-3 and TM domains are underlined. The amino acids were aligned using the DIALIGN2.1 Program (B. Morgenstern, et al. 1996). family proteins (Figure 2). It also possesses a potent carboxy terminal transmembrane domain (from residues 184 to 214) characteristic of many Bcl-2 and BNIP3 family proteins.

Interestingly, although the DNA sequence of 5'- and 3 '-untranslated regions of BNIP3h were different from other BNIP3 families, the comparison of amino acid sequence showed that BNIP3h has the same amino acid sequence with BNIP3L, NIX, and BNIP3 (Figure 2).

\section{Tissue distribution of BNIP3h}

The tissue distribution of BNIP3h mRNA in different human tissues was determined by Northern blot analysis. Two transcripts of 1.6 and $3.9 \mathrm{~kb}$ bands were detected when hybridized to multiple tissue human blot with $2.1 \mathrm{~kb}$ B764 clone as probe. These two transcripts were ubiquitously expressed in all tissues examined. BNIP3h mRNA was highly expressed in brain, heart, thymus and most abundantly in testis. A lower level of expression was detected in the liver, skeletal muscle and pancreas (Figure 3).

The expression pattern of BNIP3h was also determined in different human cancer cell lines. Human cancer cell line poly $\mathrm{A}^{+} \mathrm{RNA}$ blots (Clontech) was hybridized with a $\mathrm{P}^{32}$ labelled BNIP3h probe. As shown in Figure 3C, BNIP3h expressed in same two transcripts of 1.6 and $3.9 \mathrm{~kb}$ in all cancer cell lines examined but a lower level of expression in promyelocytic leukemia HL60, and Hela cell S3. However, the expression level was higher in chronic myelogenous leukemia K-562 and lympho-
A

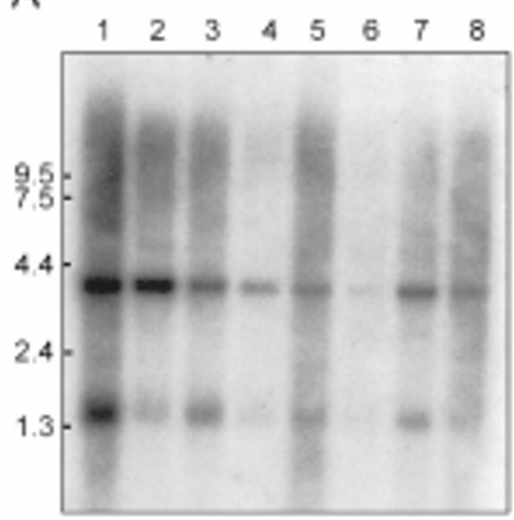
1. Heart
2. Brain
3. Palcenta
4. Lung
5. Liver
6. Skcletal muscle
7. Kidney
B. Pancreas

B

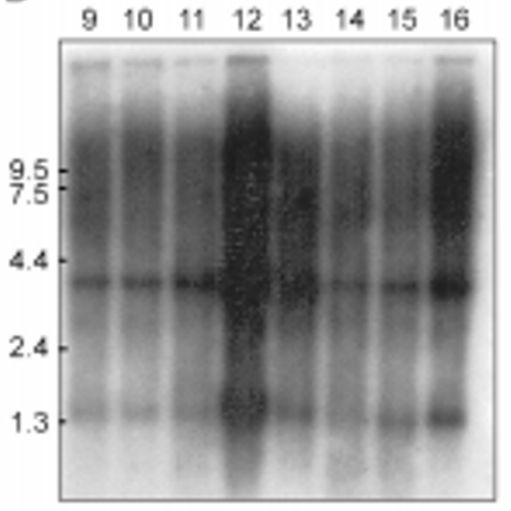

9. Spleen

10. Thymus

11. Prostate

12. Testis

13. Ovary

14. Small intestine

15. Colon

16. Peripheral blood leukocyle
C

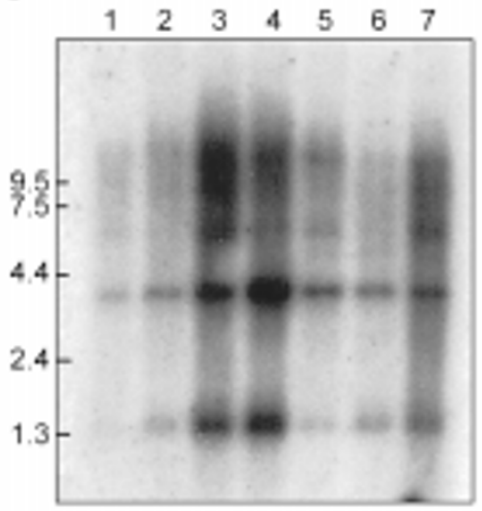

1. Promyelocytic leukemis $\mathrm{HL}-60$

2. Hela coll $\mathrm{S3}$

3. Chronic myelogenous leukemia K-562

4. Lymphoblastic leukemia Molt-5

5. Burkitt's lymphoma Raj

6. Colorectal adenocarcinoma SW480

7. Lung carcinoma A549

Figure 3. Expression of BNIP3h in human tissues and cancer cell lines. Multiple tissue northern blots (Clontech) with $2 \mu \mathrm{g}$ of mRNA from several selected adult human tissues ( $A$ and $B$ ) and cancer cell lines $(C)$ were hybridized with radiolabelled BNIP3h cDNA. The position of size markers is indicated in kilobases. 


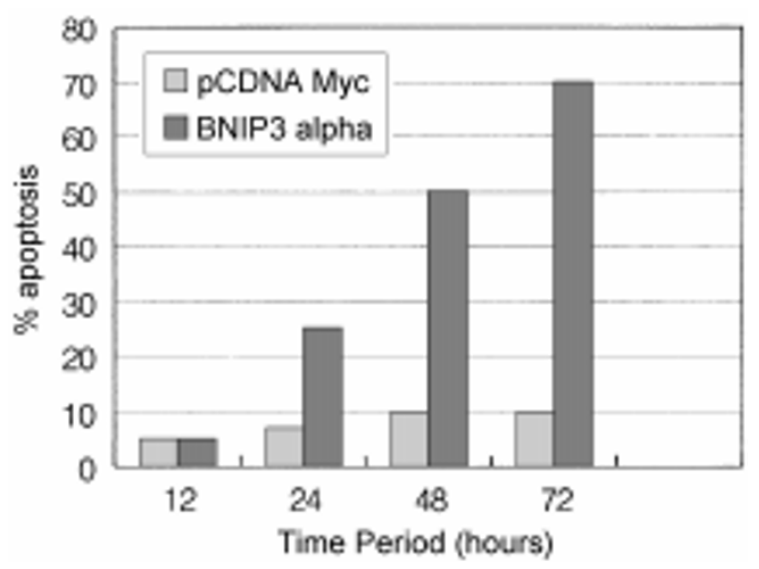

Figure 4. Proapoptotic activity of BNIP3h. MCF7 cells were transiently transfected either with $\mathrm{pcDNA} / m y c$-His (empty), or $\mathrm{pcDNA} / m y c$-His BNIP3h. The cells were fixed after $24 \mathrm{~h}, 48 \mathrm{~h}$ or $72 \mathrm{~h}$ and stained with $\beta$-Gal, and live and apoptotic cells were counted as described in Materials and Methods.

blastic leukemia MOLT-4. The identification of two transcripts suggests that the BNIP3h mRNA may be expressed as two alternatively spliced form or hybridized to other BNIP3 family genes.

\section{Pro-apoptotic activity}

About 20 to $25 \%$ of BNIP3h transfected MCF7 cells were observed as apoptotic after $24 \mathrm{~h}$. And the percentage of apoptotic cell is increased to $70 \%$ after $72 \mathrm{~h}$ (Figure 4).

\section{Discussion}

BNIP3h is a member of proapoptotic Bcl-2 family protein having homology with other BNIP3 family proteins. BNIP3h contains a $\mathrm{BH}-3$ domain which is more closely related to BNIP3, BNIP3, BNIP3L and Nix proteins than other $\mathrm{BH}-3$ containing Bcl-2 family proteins such as Bik (Boyd et al., 1995), BID (Wang et al., 1996), Hrk (Inhora et al., 1997) and BAD (Yang et al., 1995). Most BH-3 containing proapoptotic proteins induce rapid cell death when overexpressed, however, as shown by Yasuda et al. (1998a) and Figure 4 in this study, BNIP3 and BNIP3h exhibited delayed proapoptotic activity. The substantial level of apoptosis was observed $48 \mathrm{~h}$ after transfection in MFC7 cells (Figure 4). It seems that these proteins do not possess a potent proapoptotic activity like other $\mathrm{BH}-3$ containing proapoptotic proteins.

The sequence comparison of BNIP3 and BNIP3h indicates that there are several homologs of these genes, like BNIP3L (Matsumhima M. et al., 1998), BNIP3 (Yasuda M. et al., 1999), and Nix (Chen G. et al., 1999). The proteins encoded by the BNIP3L, Nix and BNIP3 genes had same amino acid sequences with BNIP3h (Figure 2). In a recent report (Chen G. et al., 1999) Nix and
$B N I P 3 L$ cDNAs encode the same amino acids sequences but they have different chromosomal locations. BNIP3L have been mapped to chromosome 8p21 and Nix to chromosome 14 (14q11.2-q12). The conceptual amino acids sequence encoded by the BNIP3h cDNA is also identical to these two proteins but it has different nucleotides sequences at both $5^{\prime}$ - and $3^{\prime}$-untranslated region. This supports the idea to have a unique name for the cDNA, which we have identified from human dermal papilla cells, as it is not the long form of any of BNIP3 family proteins. The chromosomal mapping of $B N I P 3 h$ needs to be investigated.

Amino acids sequence analysis of BNIP3h has shown that residues from 184 to 213 (Figure 2) at carboxyl terminal are similar to the transmembrane domain of $B N I P 3$. Previous indirect immunofluorescence analysis revealed that BNIP3 and its homologs were primarily localized in mitochondria (Yasuda et al., 1999, Matsushima M. et al., 1998, Chen G. et al., 1997) and carboxyl terminal transmembrane domain was thought to be responsible for the subcellular localization of these proteins. As BNIP3h possesses a transmembrane domain similar to other BNIP3 family proteins, so it may also be localized to mitochondria. It was also shown (Imazu. et al., 1999) that BNIP3L directly targets the mitochondria to induce apoptosis-associated mitochondrial changes including membrane potential loss and cytochrome C release.

Another attractive mechanism to regulate dimerization of $\mathrm{Bcl}-2$ family members is phosphorylation (Gajewski and Thompson, 1996). For example, Bad, a proapoptotic member of the Bcl-2 family, is phosphorylated by a putative kinase that can be activated by growth factor engagement (Zha et al., 1996). The phosphorylated Bad loses the ability to bind Bcl-xL. Instead, it binds to 14-33 , a protein that can interact with several signaling enzymes. The Bcl-xL dissociated from Bad, now can execute its antiapoptotic function. The possible mechanism for the induction of apoptosis by BNIP3h may be the phosphorylation by a putative kinase. As BNIP3h is rich in Ser/Thr residues, raising the possibility that activities of these proteins may be regulated by phosphorylation in response to apoptotic signals.

$B N I P 3 h$ expressed ubiquitously in all normal and cancerous human tissues examined as two transcripts of 1.6 and $3.9 \mathrm{~kb}$ (Figure 3). These results suggest that in certain human tissues BNIP3h is differentially expressed, and thus may contribute to apoptosis with some degree of specificity.

In this study, BNIP3h, a homolog of BNIP3 family protein, has been identified and functionally characterized. $B N I P 3 h$ encodes a proapoptotic protein and induces apoptosis in transfected cells. A possible direct binding activity of BNIP3h with E1B-19k and Bcl-2 and suppression of their antiapoptotic activity needs to be investigated. Among the various proapoptotic genes that have 
been identified so far, the BNIP3, BNIP3h, BNIP3, $B N I P 3 L$, and Nix genes seem to be the first examples of human proapoptotic proteins that are homologous, underscoring the fact that these proteins may play a concerted role in human apoptosis pathway.

\section{References}

Altschul SF, Gish W, Miller W, Myers EW, Lipman DJ. Basic local alignment search tool. J Mol Biol 1990;5;215:403-10

Boyd JM, Malstrom S, Subramanian T, Venkatesh LK, Schaeper U, Elangovan B, D'Sa-Eipper C, Chinnadurai G. Adenovirus $\mathrm{E} 1 \mathrm{~B} 19 \mathrm{kD}$ and $\mathrm{Bcl}-2$ proteins interact with a common set of cellular proteins. Cell 1994;79:341-51

Boyd JM, Gallo GJM, Elangovan B, Houghton AB, Malstrom S, Avery BJ, Ebb RG, Subramnian T, Chittenden T, Lutz RJ, Chinnadurai G. Bik, a novel death- inducing protein shares a distinct sequence motif with $\mathrm{Bcl}-2$ family proteins and interacts with viral and cellular survival promoting proteins. Oncogene 1995;11:1921-28

Chen G, Ray R, Dubik D, Shi L, Cizeau J, Bleackley RC, Saxena S, Gietz RD, Greenberg AH. The E1B 19K/Bcl-2binding protein Nip3 is a dimeric mitochondrial protein that activates apoptosis. Exp Med 1997;186:1975-83

Chen G, Jeannick C, Christine V, Jae H, Gracjan B, James B, Lianfa S, Don D, Arnol G. Nix and NIP3 form a subfamily of proapoptotic mitochondrial proteins. J Biol Chem 1999;274: 7-10

Chinnadurai G. Adenovirus $2 \mathrm{lp}+$ locus codes for a $19 \mathrm{kd}$ tumor antigen that plays an essential role in cell transformation. Cell 1983;33:759-66

Gajewski TF, Thompson CB. Apoptosis meets signal transduction elimination of a BAD influence. Cell 1996;87:589-92

Imazu T, Shigeomi S, Shinji T, Mieko M, Yusuke N, Tsuneharu M, Akihiko O, Yoshihide T. Bcl-2/ E1B 19 kD-interacting protein (BNIP3L) interacts with $\mathrm{Bcl}-2 / \mathrm{Bcl}-\mathrm{xl}$ and induces apoptosis by altering mitochondrial membrane permeability. Oncogene 1999;18:4523-29

Inohara N, Ding LY, Hen S, Nunez G. Harakiri, a novel regulator of cell death, encodes a protein that activate apoptosis and interact selectively with survival promoting Bcl-

\section{2 and Bcl-X(L). EMBO J 1997;16:1686-94}

Kozak M. An analysis of vertebrate mRNA sequences: initiations of translational control. J Cell Biol 1991;115:887-903

Lee JE, Sohn JW, Lee JH, Lee KC, Son CS, Tockgo YC. Regulation of bcl-2 family in hydrogen peroxide-induced apoptosis in human leukemia HL-60 cells. Exp Mol Med 2000;32:42-46

Lee JH, Park BJ, Park JH, Yang MH, Chi SG. TGF- $\beta$ inhibition of apoptosis through the transcriptional up-regulation of Bcl$\mathrm{XL}$ in human monocytic leukemia U937 cells. Exp Mol Med 1999;31:126-33

Matsushima M, Fujiwara T, Takahashi E, Minaguchi T, Equchi Y, Tsujimoto Y, Suzumori K, Nakamura Y. Isolation, mapping, and functional analysis of a novel human cDNA (BNIP3L) encoding a protein homologous to human NIP3. Genes Chromosomes Cancer 1998;21:230-35

Morgenstern B, Dress A, Werner T. Multiple DNA and protein sequence alignment based on segment-to-segment comparison. Proc Natl Acad Sci USA 1996;93:12098-103

Reed JC. Double identity of proteins of the Bcl-2 family. Nature (Lond.) 1997;387:773-76

Wang K, Yin X, Chao DT, Milliman CL, Korsmeyer SJ. BID: a novel BH-3 domain only death agonist. Genes Dev 1996;10: 2859-69

Yang E, Zha J, Jockel J, BoiseL H, Thompson CB, Korsmeyer SJ. Bad, a heterodimeric partner for $\mathrm{Bcl}-\mathrm{XL}$ and $\mathrm{Bcl}-2$ displaces Bax and promotes cell death. Cell 1995;80:285-91

Yasuda M, Theodorakis P, Subramanian T, Chinnadurai G. Adenovirus E1B-19K/BCL-2 interacting protein BNIP3 contains a $\mathrm{BH}-3$ domain and a mitochondrial targeting sequence. $\mathrm{J}$ Biol Chem 1998a;273:12415-21

Yasuda M, D'Sa-Eipper C, Gong XL, Chinnadurai G. Regulation of apoptosis by a Caenorhabditis elegans BNIP3 homolog. Oncogene 1998b;17:2525-30

Yasuda M, Han JW, Dionne CA, Boyd JMm Chinnadurai G. BNIP3alpha: a human homolog of mitochondrial proapoptotic protein BNIP3. Cancer Res 1999;59:533-37

Zha J, Harada H, Yang E, Jockel J, Korsmeyer SJ. Serine Phosphorylation of death agonist BAD on response to survival factor. Cell 1996;87:619-28 